\title{
The Role of Spatial Coherence for the Creation of Atom Size Electron Vortex Beams
}

Darius Pohl ${ }^{1,2}$, Stefan Löffler ${ }^{4}$, Sebastian Schneider ${ }^{2,3}$, Peter Tiemeijer $^{5}$, Sorin Lazar ${ }^{5}$, Kornelius Nielsch $^{2}$ and Bernd Rellinghaus ${ }^{1,2}$

1. Dresden Center for Nanoanalysis, TU Dresden, Dresden, Germany.

2. IFW Dresden, Dresden, Germany.

3. Institute for Solid State Physics, TU Dresden, Dresden, Germany.

4. University Service Centre for Transmission Electron Microscopy, TU Wien, Wien, Austria.

5. Thermo Fisher Scientific, Eindhoven, The Netherlands.

Recently discovered electron vortex beams, which carry quantized orbital angular momenta (OAM) L, promise to reveal magnetic signals [1]. Since electron beams can be easily focused down to sub-nanometer diameters, this novel technique promises the possibility to quantitatively determine local magnetic properties with unrivalled lateral resolution. To generate the spiralling wave front of an electron vortex beam with an azimuthally growing phase shift of up to $2 \pi$ and a phase singularity in its axial centre, specially designed apertures are needed [2].

In order to produce single electron vortex beams, we recently developed a method which allows to generate userdefined OAM beams [3]. We implemented two types of apertures, a $50 \mu \mathrm{m}$ fork-type aperture and a $100 \mathrm{~nm}$ small blocking aperture into the condenser lens system of a FEI Titan ${ }^{3}$ 80-300 transmission electron microscope (TEM) equipped with a probe spherical aberration corrector and a Wien-type monochromator. The selected focused probe is used for the acquisition of HRSTEM images and local EELS maps. Although today, atom size electron vortex beams can be readily generated and atomically resolved HRSTEM and EELS data can be acquired with OAMselected EVBs, the application as a probe for local measurements of the electron energy loss chiral dichroism (EMCD) suffers from a poor $\mathrm{S} / \mathrm{N}$ ratio and is thus still under development. One reason may be the incoherence of the vortex beam itself, which results in a strong reduction of the dichroic signal. Measurements on ferrimagnetic samples reveal magnetic signals, which are by far smaller than expected from simulations.

The monochromator provides for the flexibility to tune the degree of coherence on the vortex aperture by allowing to continuously vary the gun lens (GL) excitation. The coherence of the electron beam plays an important role for the purity of the OAM of the generated electron vortex beam. In this work we have therefore investigated the influence of the gun lens excitation on the coherence of the electron vortex beam. As a measure of this coherence, the central intensity dip of the imaged probe is determined (see fig1) [5]. Images of the vortex probe have been acquired utilyzing two types of electron guns: (i) a standard Shottky FEG (SFEG) and (ii) a so-called highbrightness gun (XFEG). Normalized rotationally averaged profiles of the images are used to analyse the dependency of the EVB quality on the coherence (gun lens excitation). Fig. 2 summarizes the results. Both electron guns show an improvement of the spatial coherence with increasing gun lens excitation, measured by the central intensity dip in the $|\mathrm{L}|=1 \hbar$ vortex beam. For the XFEG operated at $200 \mathrm{kV}$, a central intensity dip of $\mathrm{L}_{\text {Dip }} / \mathrm{I}_{\max }=0.3$ can be reached. Using the dip value and taking into account the size of the aperture and the convergence semi-angle of $22.5 \mathrm{mrad}$, we calculated the incoherence angle $\alpha_{\text {incoh }}$ to be between $45 \mathrm{nrad}$ and $25 \mathrm{nrad}$ for a gun lens excitation from $6 \mathrm{~V}$ to $200 \mathrm{~V}[3,4]$. Following the van Cittert-Zernike theorem, the coherence length $\mathrm{d}_{\mathrm{C}}$ in the aperture plane can be calculated from:

$\mathrm{d}_{\mathrm{C}}=\lambda / \pi \alpha_{\text {incoh }}$,

which results in $\mathrm{d}_{\mathrm{C}}=32 \mu \mathrm{m}$ (for the $200 \mathrm{kV}$ XFEG, GL $=200 \mathrm{~V}$ ) that is thus substantially smaller than the diameter of the fork aperture. 
Hence, EVBs generated from fully coherent primary beams may by the missing piece to the puzzle as to how to substantially improve quantitative magnetic signals as obtained from individual atomic rows.

\section{References:}

[1] J. Verbeeck et al., Nature 467 (2010), p. 301.

[2] J. Verbeeck et al., Ultramicroscopy 113 (2012), p. 83.

[3] D. Pohl et al., Sci. Rep. 7 (2017), p. 934.

[4] P. Schattschneider et al., Ultramicroscopy 111 (2011), p. 1461.

[5] P. Schattschneider et al., Ultramicroscopy 115 (2012), p. 21.
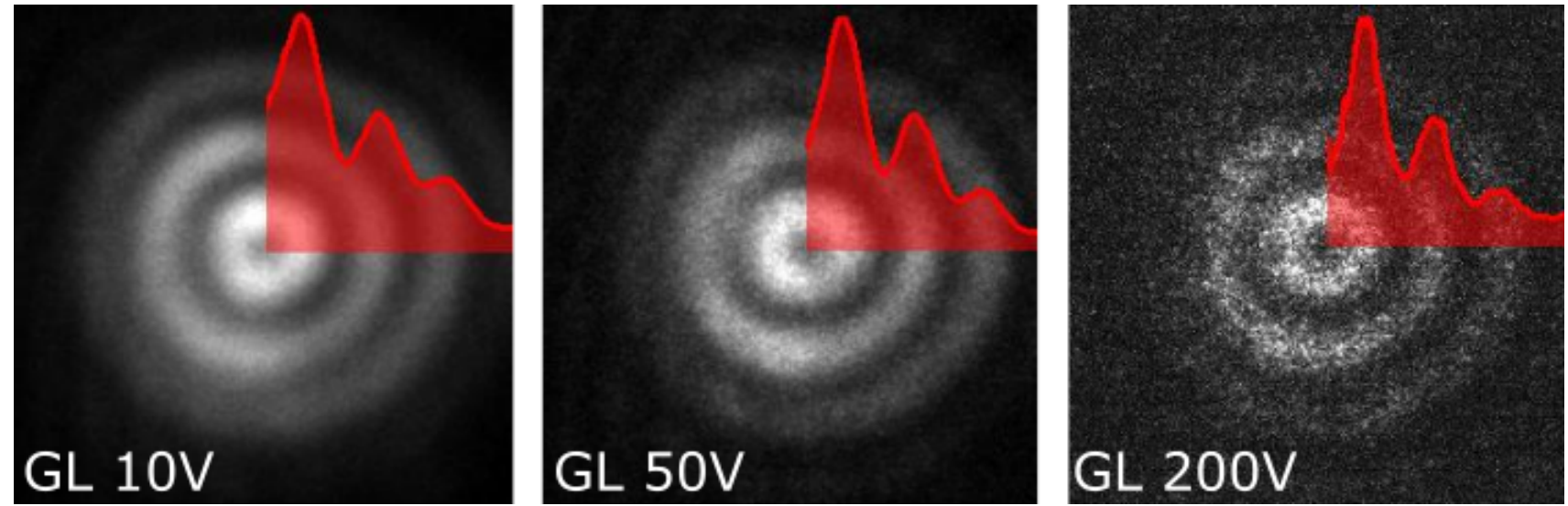

Figure 1. Images of the electron vortex beam for different gun lens excitations using a XFEG @ 200kV. An exposure time of $0.5 \mathrm{sec}$ was used for all images. Insets show normalized and rotationally averaged intensity profiles.

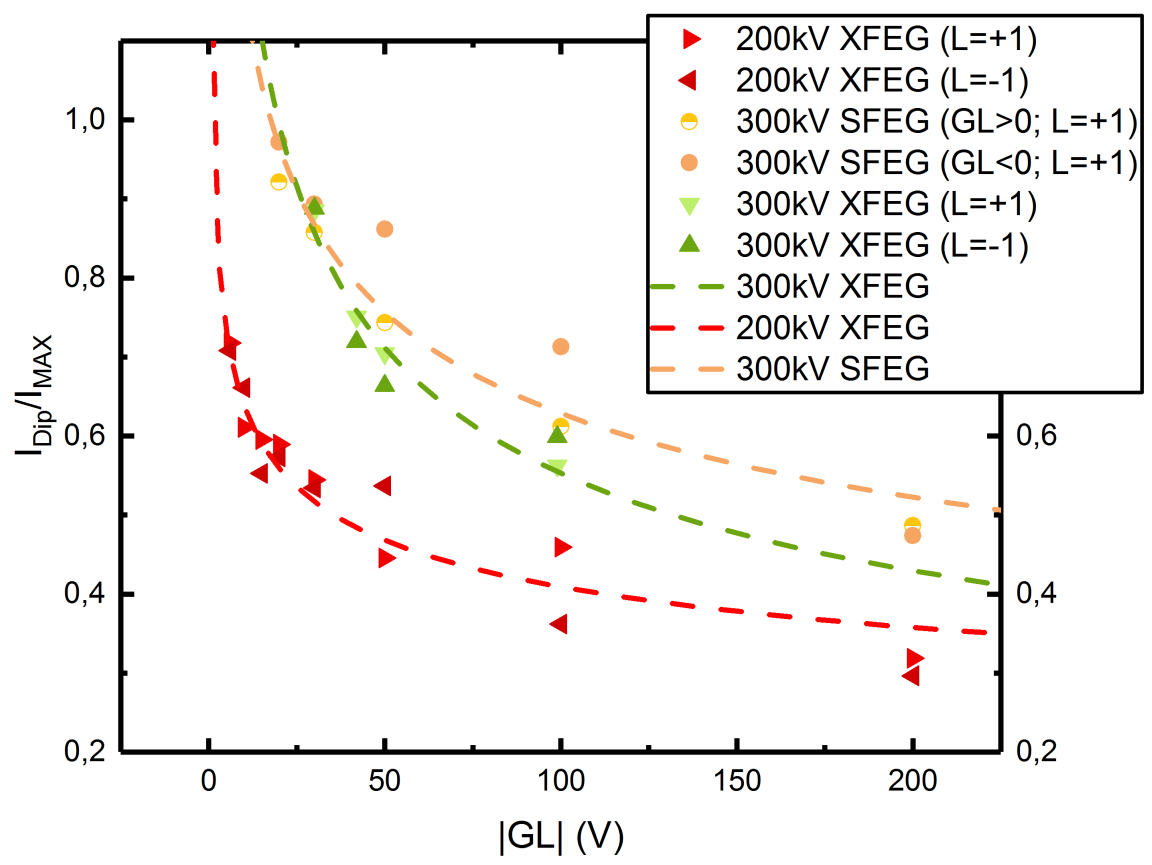

Figure 2. Relative intensity of the local minima at the center of the EVB as a function of the gun lens excitation for a SFEG and a XFEG system. 